\title{
Stability and Complexity in Digital Ecosystems
}

\author{
Paul J. Krause, Fellow, IMA, Amir R. Razavi, Sotiris Moschoyiannis, Member, IEEE, and Alexandros Marinos
}

Department of Computing, Surrey University, Guildford, GU2 7XH, UK

e-mail: (p.krause, a.razavi, s.moschoyiannis ,a.marinos)@surrey.ac.uk

\begin{abstract}
In this paper we explore the concept of "ecosystem" as a metaphor in the development of the digital economy. We argue that the modelling of social ecosystems as selforganising systems is also relevant to the study of digital ecosystems. Specifically, that centralised control structures in digital ecosystems militate against emergence of innovation and adaptive response to pressures or shocks that may impact the ecosystem. We hope the paper will stimulate a more holistic approach to gaining empirical and theoretical understanding of digital ecosystems.
\end{abstract}

Index Terms-digital ecosystems, self-organisation, collaborative working, social-ecosystems, digital economy.

\section{INTRODUCTION}

Although we have used a modification of the title of Lord May's landmark treatise in theoretical ecology [15], we do not intend any comparison to be made with regard to the potential impact of this paper. Rather, we use the title to highlight the importance of progressing from the use of the term "Ecosystems" as a metaphor in the study of Digital Ecosystems, to the development of a theoretical foundation of Digital Ecosystems. In this paper we will explore a number of theoretical and empirical advances in the study of natural- and social-ecosystems, and draw out a number of hypotheses that can be explored to assess their relevance to the study of digital ecosystems.

Boley and Chang [2] proposed a definition of a digital ecosystem, based on analogy with their own definition of ecosystem in the purely biological sense. In their view, a digital ecosystem was:

"an open, loosely coupled, domain clustered, demanddriven, self-organising agent environment, where each agent of each species is proactive and responsive regarding its own benefit/profit (...) but is also responsible to its system."

A key feature of this is that agents join freely and of their own volition. This is in contrast to a tightly coupled organisation in which the agents have pre-defined roles. The focus is very much on the autonomy of individual agents, and hence the global properties and institutions of such an ecosystem emerge (primarily) through self-organisation. This is a radical and potentially disruptive concept. However, we will argue later in this paper that current digital infrastructures constrain the emergence of properties and institutions, and instead impose such from outside the ecosystem.

To this end, we would like to work from a definition of an ecosystem that is at a higher level of abstraction. This is purely for the purposes of the discussion in this paper, and not intended as an alternative to the above definition, which we fully support.

We take as our starting point the Arthur Tansley's 1935 definition of an ecosystem as, [24]:

"An interactive system established between living creatures and the environment in which they live."

With regards to a digital ecosystem, we want to hold back any discussion around "living" for the moment, and so work with the following:

"A digital ecosystem is an interactive system established between a set of active agents and an environment within which they engage in common activities."

Note that, consistent with this definition, it is possible for a given agent to interact in multiple digital ecosystems. "Agents" include (but might not be limited to) providers of software services, information sources, and human agents. The environment is a combination of a socio-economic context and a digital infrastructure. We will argue that the nature of the latter, the digital infrastructure, can impact (undesirably at present) on the properties that emerge in the ecosystem.

Our view is that the concept of species, even in natural ecosystems, has some difficulties. Nevertheless, it is difficult to engage in any dialogue on ecosystems without some reference to species, and it can be useful of course. Boley and Chang referred to species as "types of agents" [2]. We concur with that, but it still leaves open the question of how we define a taxonomy for digital ecosystems?

We don't want to address that question in this paper. However, we would like to work with a fragment of a taxonomy for the purposes of discussion. In commerce, a business enterprise can be viewed as providing a set of services. In that such an enterprise provides a higher level of organisation to an individual service, we might consider (business) enterprises as species within an ecosystem. This equating of enterprise with species will be particularly useful when we come to discuss response diversity in digital ecosystems.

In the next section we illustrate the importance of innovation processes in sustaining ecosystems. After that we introduce some key concepts in ecological modelling, following [12]. We then outline some of the issues with current digital ecosystems before returning to the modelling of social-ecosystems. Finally, we conclude with some pointers to a research agenda for the science of digital ecosystems. 


\section{THE K-T EXTINCTION EvENT}

The non-avian Dinosaurs and certain marine creatures were subject to a massive extinction event at the Cretaceous-Tertiary (K-T) boundary, about 65 Million Years ago. The current preferred theory seems to be that this was triggered by a massive meteorite impact at Chicxulub in what is now North-east Mexico. There is little doubt that this meteorite impact did cause widespread disruption to the global environment. However, it is less clear that this was the sole "impactor" on the inhabitants of the earth. Even more importantly, evidence is emerging that this did not even coincide with a period in which there was a net loss of biodiversity on the planet. Rather, the reductions in certain populations were being countered by ongoing speciation activities in other groups of species.

The first point to note is that the meteor impact was not the only major event that was effecting the physical environment of the time. In addition, the K-T boundary of 65 million years ago marked the final stages of the break up of the supercontinent Pangea into the broad continental structure that is now seen. This was a gradual process that started some 225 million years ago. The period from 135 million years ago (Jurassic Park time!) to 65 million years ago saw the separation of the plates that form North America and Eurasia, and the plates that form South America, Antarctica and Australasia. As well as this fragmentation of what were formerly massive continental areas, the continuing plate movements were beginning to form many of the topological features (mountains and high altitude plateaus) that are now homes to large pools of endemic species.

As an example, the huge sauropod footprints that were discovered in Croatia by a team led by Michael Caldwell of the University of Alberta (but published by Mezga et al, Cretac. Res. 27, 735-742, 2006 - such is the cutthroat world of scientific research!) were laid down about 95 million years ago. As well as the important paleontological significance, these also represent the most recent signs of life on what is known as the Adriatic-Dinaric platform before is sunk below sea level 94 million years ago. This is clear evidence of niche removal for certain dinosaur species in one specific area.

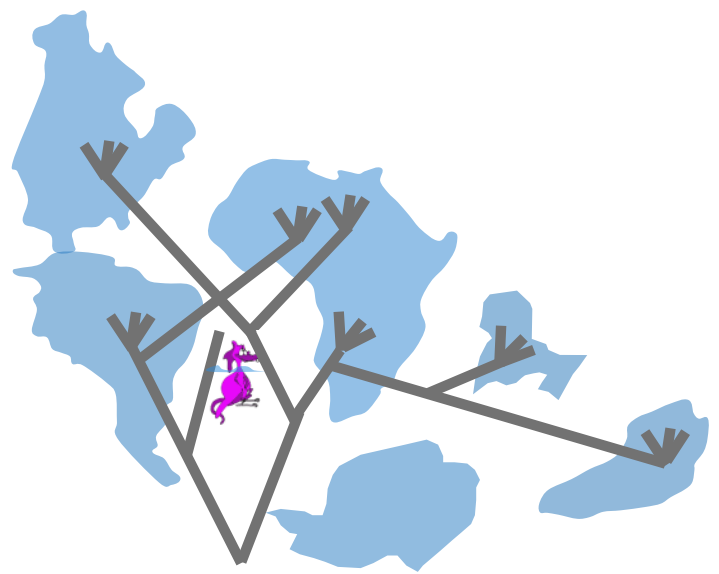

Fig.1 A major speciation event occurred prior to the dinosaur extinction, perhaps triggered by continuing changes in the Earth's geomorphology.

Simultaneous to this niche destruction process that ap- pears to have provided an adverse selection pressure on non-avian dinosaurs, recent evidence indicates that there was on going speciation amongst both mammals [1] and the avian fauna [3]. Bininda-Emonds et al, [1] have constructed a near complete species phylogeny for the extant mammals. This includes estimates of divergence times using a combination of gene alignment techniques and fossil calibration points. A key result was their identification of two periods of diversification. The first was from 100-85 Million years ago. Again note that this coincide with the final stages of the break up of the supercontinental landmasses, although at the moment this is only a statement of coincidence and needs further theoretical investigation. The second appeared in the early Eocene, yet at approximately 50 millions years ago this was significantly after the K-T boundary. A key conclusion of [1] is that this "challenges the hypothesis that the end-Cretaceous mass extinction event had a major influence on the diversification of today's mammals".

Our proposal is that the K-T extinction event was a (final) perturbation in a long-term species innovation process that was driven by the continued break up and change to the topologies of the supercontinental landmasses. By the time of the final extinction of the dinosaurs, landmasses were significantly fragmented, separated by large bodies of water and undergoing significant topological changes due to tectonic activity. Overall, this led to acceleration in the Birth Death Innovation Model that underpins the evolution of biodiversity on this planet. Finally, and most importantly in terms of its relevance to our claims about the importance of support for SMEs in a DE, the long tail of rare species played a critical role as a pool of innovation during this adaptation of the natural ecosystem in response to this longterm period of niche creation and destruction.

\section{MODELS OF SOCIAL-ECOSYSTEMS}

A primary goal behind our studies of ecological modelling for digital ecosystems is the avoidance of premature commitments to specific technological solutions or directions; the art of engaging with new communities is to leave your baggage and preconceptions behind. Instead, we will adhere to two guiding assumptions:

Firstly, our core research should focus on generative and disruptive, rather than "sterile", technologies;

Secondly, minimizing the imposition of centralised control or organisation will enhance the ability of the Digital Economy to empower or transform a given socio-economic context.

The two assumptions are closely related, and could be summarised as a target of transforming the Digital Economy into a complex adaptive system, where institutions emerge and evolve through local interactions and memory.

These are, of course, assumptions. Their validity must be established through both empirical and theoretical studies. 


\section{A. Adaptive Cycles, Resilience and Trans- formation}

The concept of "ecosystem" is increasingly being used as a metaphor in business and systems thinking. In itself, this is a more inclusive view than the more traditional hierarchical, or value-chain models - it provides greater acknowledgement of the importance of a community as a whole in sustaining value creation. However, one can go much further in using the metaphor to drive the development of models that have real value in understanding and facilitating transformation in the Digital Economy.

The adaptive cycle concept emerged from studies of regional development and ecosystem management that were conducted in the 1980's and 90's [11]. The concept of a climax community is well understood in natural ecosystems. This corresponds to the $K$ or Conservation phase of the adaptive cycle in Figure 1. But established business or stable socio-economic ecosystems also have an analogous $\mathrm{K}$ phase in which interventions and controls are typically chosen to maintain or streamline the delivery of the ecosystem's outputs.

At some time, a change in either an internal or an external variable may trigger a transformation to the $\Omega$ or release phase (e.g. a forest fire, in a natural system, or the shift from a product oriented to a service oriented market place in the case of business ecosystems). The system may then respond with reorganisation before moving back through exploitation into a new (and possibly qualitatively different) conservation phase.

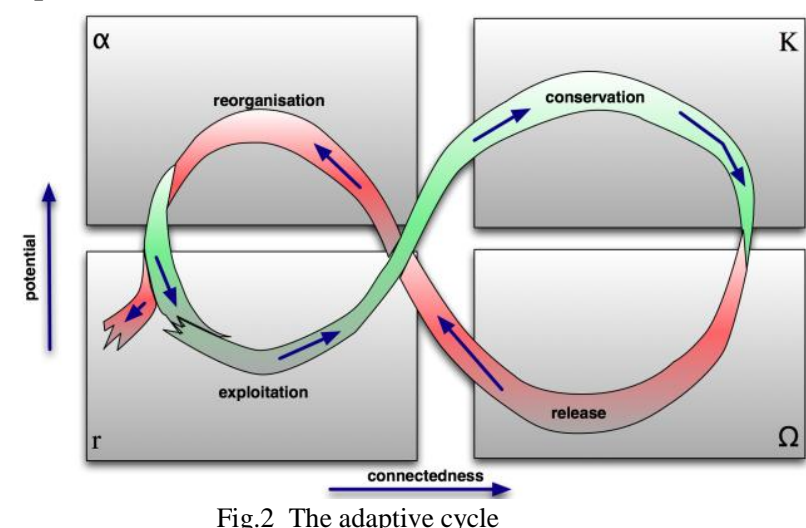

The above adaptive cycle works well as a metaphor for many social-ecological systems. It also provides a useful framework for posing research questions and structuring simulations/experiments in Digital Ecosystems [12].

An important extension of this is that in most systems there is a hierarchy of such cycles (usually working on different timescales). A change in system variables in one level can trigger change in other levels. Typically a large business will hope to work in " $\mathrm{K}$ " sector. But a change in the higherlevel business environment can trigger transition to $\Omega$ and then $\alpha$. Note that if the response is not effective, the cycle can exit at "r".

Both natural and social/business ecosystems will demonstrate a degree of resilience to external change or shocks whilst in the $K$ sector. In part a certain level of response diversity in the face of external change can provide this. Con- trast the coral reefs of Great Barrier Reef (GBR) to those of the Caribbean. There is much higher species diversity in GBR. This in turn means that the various (trophic) functional groups in GBR have significantly enhanced response diversity compared with the coral reef ecosystem in the Caribbean. Broadly, this is what is currently providing the GBR with greater resilience than the coral reefs of the Caribbean (where there has been an $80 \%$ decline in hard corals).

This metaphor of response diversity in business ecosystems suggests that SMEs are a major factor in maintaining resilience of the ecosystems in the face of extreme events. This hypothesis is not established, but it does raise interesting research questions that can be addressed through simulations and comparative analyses of real-world case-studies. (It is appropriate that this paper was written in a time of financial uncertainty where resilience will be tested to the full.)

In general, resilience is a property that emerges from a range of features in any complex system. The mistake is often to use simple interventions to try to control a system in the face of some external pressure or shock, without considering, or even being aware of, all the variables that impact on the stability of the state of a complex ecosystem.

A number of case studies on the resilience of a range of social-ecosystems can be found in [12]. Some key lessons can be drawn from this:

Interventions are often planned without taking into account, or even being aware of, one of the key variables of the ecosystem;

Interventions are planned on individual components of a complex system, without modelling how the system may adapt as a whole to these changes of individual components;

Ill-conceived interventions can impact on the resilience of an ecosystem to withstand extreme events;

Without carefully modelling the impact of an intervention, we may trigger an unrecoverable transition from one stable state to another (for example, the transformation of species rich sawgrass communities to single species stands of cattail, in the Florida Everglades).

In the case of business ecosystems, we believe SMEs play a critical role in maintaining the response diversity in the face of global change. However, as will be discussed shortly, SMEs are not fully engaged in the Digital Economy, and in many cases are seriously disenfranchised from it.

The importance of understanding the dynamics arising from the complexity of ecosystems and "panarchies" of ecosystems is not just in understanding how to maintain a valued ecosystem. It also helps us to understand the variables that impact on our ability to transform an ecosystem into a new, more desirable, state.

This has provided a short introduction to a number of key concepts. Before extending this to cover sustainability and the important enhancements of memory and intention that are needed for the study of social ecosystems, we will provide an overview of the current situation with digital ecosystems. 


\section{IV.AN INCLUSIVE DIGITAL ECOSYSTEM FOR SMES}

In the EU-27, the percentage of enterprises' total turnover from e-commerce via the internet doubled between 2004 and 2007, passing from 2.1\% (2004) to $4.2 \%$ (2007) of total turnover [8]. However, for B2B transactions among SMEs, on average only about $11 \%$ of SMEs use software solutions or internet-based services for e-procurement. Moreover, there is a massive gap between the percentage of SMEs placing at least some orders online (53\% of total) and those that use special software for this (only $11 \%$ of total). SMEs without special software place orders mainly through websites or extranets of suppliers [9]. The result is a lack of digital back-office integration of procurementrelated processes among European SMEs.

As discussed in [7] a major barrier to the expansion of use of B2B amongst SMEs is that the current standards for web service coordination require a central coordinator. Even where there is a natural hub for the business activities, this can provide a threat to the local autonomy of the participating SMEs - the web service coordinator will have access to business state, business logic and data about traffic that can help in enforcing a level of governance on the participating SMEs, and also inform acquisition policies of the coordinator. A distributed coordination model for longrunning business transactions that aims to alleviate such concerns has been proposed in $[17,20]$.

However, these are not the only barriers to adoption of digital innovation amongst SMEs. Our own studies in the Cambridge region indicate more fundamental issues need to be addressed, including: Intense frustration at the lack of interoperability and loss of core business time; An already low ICT take up.

This situation is frustrating a potential expansion of the digital economy that would be of major significance. The focus on networking, collaboration and cooperation continues to develop in modern business processes [14], and is especially important amongst communities of SMEs. Greater inclusion into a technology enabled business landscape will enhance their capacity for achieving a form of "competitive co-evolution" [18]. We believe this model is especially relevant to the information-based industries that are becoming increasingly important in the redevelopment of the UK economy.

The tourism industry provides an example of the strong contrasts in inclusivity of the Digital Economy [4]. Increasingly, consumers rely on the Internet to search for information, and book their vacation. Yet, while many of the more satisfying (and socio-ecologically sensitive) vacation experiences can be found and booked through "microtourism" websites, these are easily eclipsed by the megatourism sites such as Travelocity and Expedia.

The barriers to adoption are not just technological. Evans at al [10] found that:

Micro and small tourism enterprises don't identify themselves with the mainstream tourism industry;

The lifestyle choice of owner-operators often militates against their take up of ICT;
In addition, tourism SMEs tend to rely in intermediaries for their marketing and so have limited bargaining power in the distribution channel [6]. Our own work with the UK Technology Strategy Board and Regional Development Agencies indicates that these problems arise repeatedly across many SME sectors.

The key lesson we want to draw from the above is that the development of digital ecosystems needs to take into account the social perspectives of its members. To that effect we now return to ecological modelling and expand this to discuss inclusion of the socio-economic context in the models.

\section{RESPONSE AND SUSTAINABILITY}

So far, we have emphasised the importance of response diversity in providing a degree of resilience to shocks on an ecosystem. However, this may not be sufficient to guarantee recovery of an ecosystem. In addition, it is not only shocks that may trigger the transition of an ecosystem from the $\mathrm{K}$ (conservation) phase to the $\Omega$ (release) phase of the adaptive cycle, and its subsequent reorganisation into a qualitatively different regime. There is good empirical evidence from natural ecosystems that in certain circumstances they can respond catastrophically to a gradual increase in stress.

\section{A. Catastrophic responses}

We will summarise the discussion from Scheffer et al. in this sub-section [23]. For simplicity we will only consider one ecosystem state variable, and one impacting state variable. A common assumption in ecosystem management is that some increase in stress on that ecosystem will lead to a steady and gradual change in its state (figure 3 , below).

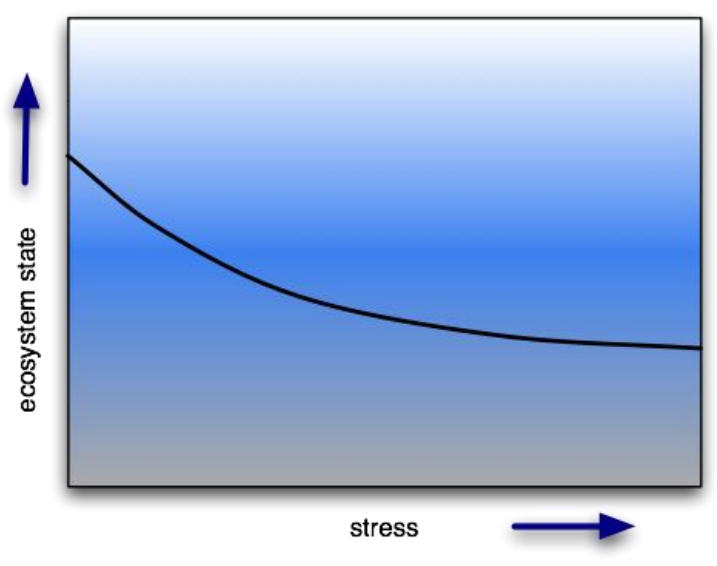

Fig.3 Naive model of ecosystem response to stress

This supports a comfortable feeling that if the ecosystem state deteriorates below an acceptable level for certain stakeholders, then it may be recovered simply by reducing the stress. That is, a $\mathrm{K}$ to $\Omega$ transition may be reversible.

Unfortunately, as we will describe in a concrete example shortly, a given ecosystem may support (at least) two stable equilibrium states over a range of environmental conditions. In such a scenario, the ecosystem may be relatively inert to change in stress over a certain range of conditions, but then respond with a catastrophic switch to a new stable state 
once the environmental stress exceeds a certain threshold (point $\mathrm{F}_{2}$ in figure 4 above).

Two key issues arise. Firstly, given the relatively benign initial response of the ecosystem to the environmental stress, the catastrophic switch to a new stable state will typically be hard to predict and prepare for. Secondly, given the hysteresis effect of such a response curve, a very significant reduction in stress level will be needed before the ecosystem will transition back to the upper branch (point $F_{1}$ in figure 4). Indeed, it can happen that the point $F_{1}$ is to the left of the origin, and hence not reachable through a simple reduction in the stress factor that lead to the transition to the lower branch.

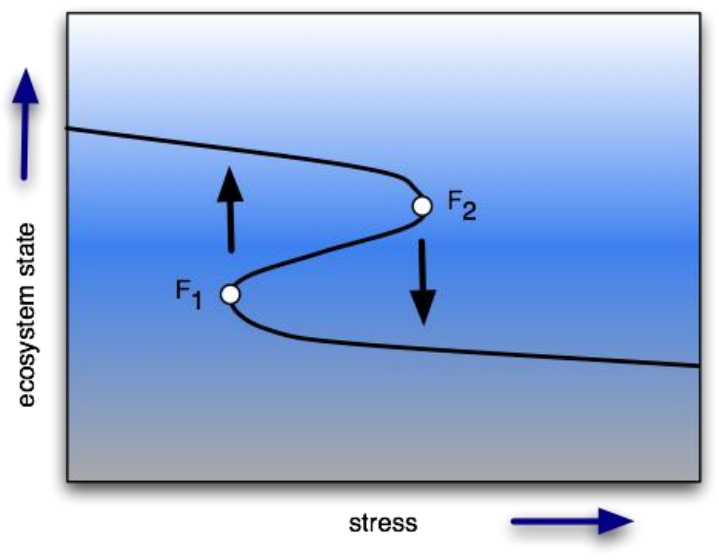

Fig.4 Stress may induce catastrophic change

It is worth including a concrete example to keep this paper self-contained. Both clear lakes, and arid ecosystems fit well to the above model, but given the greater experience of linking them into socio-economic systems, we will use the example of clear lakes following [5]. Fertiliser run-off from agricultural communities, and wastewater from other human activities can impact clear shallow lakes. The resulting increase in nutrients ("stress") stimulates the growth of phytoplankton. The resulting green turbidity reduces light intensity and hence impacts the characteristically lush plant growth on the bottom of the lake. This impacts on the small animals that feed amongst this vegetation. The fish species that feed on such animals, attach eggs to such plants or use submerged plants for shelter will then also decline. Finally, in response to the reduction in fish species, the number of birds visiting the lake will decline.

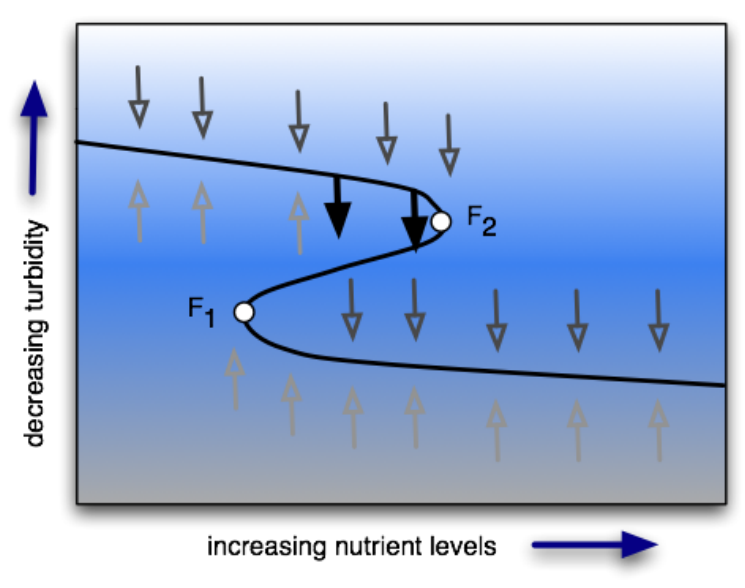

Fig.5 Resilience is minimal near the tipping points $F_{1}$ and $F_{2}$

A disturbance, or shock, to the ecosystem can be represented by a vertical displacement from the equilibrium line. Such a disturbance in this case might be a sudden increase in nutrient levels due to prolonged heavy rain, or a significant fish kill. Once the disturbance ceases, the system will return to equilibrium (the grey arrows). But note that if a large disturbance or shock occurs (represented by the heavy black arrows) near to the point $F_{2}$, it may tip the system into the second highly turbid stable state.

\section{B. Resilience and Sustainability}

It is still hard to gain a consensus on the semantics of sustainability as a concept. During the lead up to the Local Agenda 21 actions at the end of the last century, the Brundtland Commission defined sustainable development as: "development that meets the needs of present generations without compromising the ability of future generations to meet their needs" [26]. This is worthy, but a little hard to operationalise; does it mean that we should wind down oil consumption in case a future generation has a greater need for it, for example?

An alternative approach is to consider the sustainability of a social-ecosystem in terms of its capacity to absorb shocks and chronic stress [13]. The concepts of resilience and the adaptive cycle then become important in guiding studies to aid the effective monitoring and management of complex (socio-economic, digital and natural) ecosystems. Provided we can identify all the key variables in an ecosystem, we may be able to combine theoretical and empirical analyses to develop a model of its response to change. As we have seen in the previous section, such models may need to be non-linear or even chaotic [5].

The adaptive cycle reminds us that sustainability should not be equated with stasis. A natural ecosystem may have a need to periodically cycle through instances of the adaptive cycle in order to avoid moving into a situation where a small disturbance could trigger a dramatic or catastrophic switch into an alternative stable state. A well-known example of this is the impact of fire control in temperate forests. Small scale, local, fires $(\Omega$, release, phase) clear forest debris and lead to new growth, regeneration and gradual transformation back to a climax vegetation $(\alpha \rightarrow \mathrm{r} \rightarrow \mathrm{K})$. At- 
tempts to control this natural cycle lead to a widespread and deep accumulation of forest debris. The potential for a catastrophic forest fire then builds up leading to at best a release phase that is hard, costly and extremely risky for humans to control, and at worst opens the risk of transformation into a new stable state (perhaps regeneration is dominated by a non-native water greedy tree species, that locks out recovery by the previous vegetation).

Things become more complex (and more relevant to digital ecosystems) when we include human social and economic interests into the ecosystem models. At this point we will quite often (perhaps usually) move away from closed cycles around the adaptive figure of eight. The release phase may be triggered by a factor internal ("revolution") or external ("remember") to the system. The ecosystem then needs (as humans are now involved, aspects of intention become relevant) to reorganise into a different state in order to succeed in progressing through the exploitation phase into a new (quasi-stable) conservation phase.

Gunderson and Holling [12] use the example of the Florida Everglades to demonstrate the institutional response to a series of ecological crises over the last century. Essentially the tensions there are between water management to support increasing economic development in a natural ecosystem where widespread flooding (release phase) was an important part of the latter's adaptive cycle. To summarise briefly, we list here the institutions that were generated, together with the crisis that triggered the reconfiguration:

- 1903, Everglades Drainage District (Flood)

- 1947, Central and Southern Florida Flood Control District (Flood)

- 1971, South Florida Water Management District (Drought)

- 1983, Everglades Coalition (perception of switch to new ecological state and fear of pollution through high rainfall)

- 1989, Everglades National Park Protection and Expansion Act (Costly water quality lawsuit)

A full description of the evolution of the Everglades social-ecosystem can be found in [25]. The important point here is that the Florida Everglades social-ecosystem went through a sequence of "release" and reorganisation phases as memories of the consequences of external shocks triggered the emergence of new institutions. These can be thought of as representative of new states in the history (ontogeny) of a complex adaptive socio-economic system. Collective memory in the system (hopefully) prevents reversion to an earlier, less resilient, state. Collective intention, in so far as it exists, helps the system to reorganise following release phases into states that are (hopefully) more resilient to external and internal tensions.

The development of well-informed models of socialeconomic systems is absolutely essential for continuing to guide the reorganisation of complex social-ecosystems following a release phase.

\section{LESSONS FOR DIGITAL ECOSYSTEMS}

The motivation behind the writing of this position paper was to raise awareness of an extensive corpus of empirical and theoretical studies of social-economic ecosystems that has shown real benefit in informing decisions in the management of such systems. Out thesis is that much of this work can form the foundation for similar studies of digital ecosystems.

We can already draw some provisional conclusions that are informing our own work and that of other partners in the OPAALS Network of Excellence [20,21]. We have seen that a key impactor on resilience of an ecosystem is the ability of the species that interact in that system to be able to maintain key functions as conditions vary [19]. The concern with current digital ecosystems is that they are often, perhaps always, focused around a single or a small number of centralised hubs. This provides a fundamental limitation on the response diversity of digital ecosystems. As with any natural ecosystem [13], we believe that the "functional diversity of species that support critical structuring processes" [5] is critical to the resilience of any digital ecosystem. Referring back to our view of enterprises as (one kind of) species within digital ecosystems, we believe that this requires us to develop digital infrastructures that respect and support the autonomy of small to medium sized enterprises (SMEs); the long tail of diversity in any resilient digital ecosystem [22].

Whilst some of the modelling from social-ecosystems may be directly relevant to digital ecosystems, there are some important distinctions too. For example, we mentioned that an agent might interact with more than one digital ecosystem. This is because in general, an agent may take multiple roles - a human agent, for example, may interact in a work related ecosystem, and an interest related ecosystem without cross-over between these roles. However, it is possible that one or more agents may identify a potential join between two previously separated ecosystems. As a concrete example, at the time of writing we are working to arrange funding to support a join between tourism and environmental/nature conservation digital ecosystems in India. This is an important form of innovation and can see the merging of two previously disjoint ecosystem ontogenies. We do not believe such merges have so far been studied.

There is a more private agenda too. We believe that effective use of digital ecosystems could lead to changes in lifestyles and working practices that could have significant environmental benefits (reduction in business travel, rural regeneration, for example). Our hope is that bringing an understanding of the dynamics of natural ecosystems into the science of digital ecosystems could help raise awareness of the importance of and lifestyle benefits to be gained by, a more holistic way of living.

\section{ACKNOWLEDGEMENTS}

This work was supported by the EU-FP6 funded project OPAALS Contract No 034824.

\section{REFERENCES}

[1] O.R.P. Bininda-Edmonds et al, "The delayed rise of present-day mammals," Nature, vol.446, 2007, 507-512. 
[2] H. Boley and E. Chang, "Digital Ecosystems: Principles and Semantics", in Proceedings of the 2007 Inaugural IEEE Conference on Digital Ecosystems and Technologies, 2007, 1-6.

[3] J.W. Brown, R.B. Payne and D.P.Mindell, "Nuclear DNA does not reconcile 'rocks' and 'clocks' in Neoaves", Biol. Lett., 2007.

[4] P. Braun, E-Commerce and Small Tourism Firms, In: Marshall S., Taylor, W. and Yu X. (eds), Encyclopedia of Developing Regional Communities with Information and Communication Technology, Idea Group, 2006.

[5] W.A. Brock, K. Mäler and C. Perrings, "Resilience and Sustainability: The Economic Analysis of Nonlinear Dynamic Systems", in: L.H.S. Gunderson and C.S. Holling, q.v., 2002, 261-289.

[6] D. Buhalis, "Information Technology for Small and Medium-Sized Tourims Enterprises: Adaptation and Benefits," Information Technology and Tourism, 2, 79-95, 1999.

[7] P. Dini, G. Lombardo, R. Mansell, A. Razavi, S. Moschoyiannis, P. Krause, A. Nicolai and L. Leon, Beyond interoperability to digital ecosystems: regional innovation and socio-economic development led by SMEs, International Journal of Technological Learning, Innovation and Development, 1:3, 410 - 426, 2008.

[8] Eurostat, Community Survey on ICT Usage and E-commerce in Enterprises, Brussels, 2007.

[9] European Commission, The European e-Business Report, eBusiness Watch, Luxembourg, 2007.

[10] G. Evans, J. Bohrer and G. Richards, "Small is Beautiful? ICT and Tourism SMEs - A comparative European Study", Information Technology and Tourism, 3, 139-153, 2001.

[11] L.H.S. Gunderson, C.S. Holling and S.S. Light (eds), Barriers and Bridges in the Renewal of Ecosystems and Institutions, Columbia University Press, New York, 1995.

[12] L.H.S. Gunderson and C.S. Holling, Panarchy: Understanding transformations in human and natural systems, Island Press, 2002.

[13] C.S. Holling, D.W. Schindler, B.W. Walker and J. Roughgarden, "Biodiversity in the functioning of ecosystems", in C.A. Perrings et al., Rights to Nature, 1995, 44-83.
[14] J. Marceau and M. Dodgson, Systems of Innovation, Proc. National Innovation Summit, Melbourne, 2000.

[15] R.M. May, Stability and Complexity in Model Ecosystems, Princeton University Press, Princeton and Oxford: 2001.

[16] M. Mazzanti and R. Zoboli, "Economic instruments and induced innovation: The European policies on end-of-life vehicles", Ecological Economics, vol.58, 2006, 318-337.

[17] S. Moschoyiannis, A. Razavi and P. Krause, "Transaction Scripts: making implicit scenarios explicit," in Proc. ETAPS 2008 FESCA'08, ENTCS, Elsevier, 2009. To appear

[18] A. Ordanini, "Infomediation and Competitive Advantage in B2B Digital Marketplace", European Management Journal, vol.19, 2001 276-285.

[19] C. Perrings, "Ecological resilience in the sustainability of economic development," Economie Appliqueé, vol.48, 1995, 121-142.

[20] A. Razavi, S. Moschoyiannis and P. Krause, "A Coordination Model for Distributed Transactions in Digital Ecosystems," in IEEE Digital Ecosystems and Technologies (IEEE-DEST'07), 2007.

[21] A. Razavi, S. Moschoyiannis and P. Krause, "A Scale-free Business Network for Digital Ecosystems," in IEEE Digital Ecosystems and Technologies (IEEE-DEST'08), 2008.

[22] A. Razavi, S. Moschoyiannis and P. Krause, "A Self-Organising Environment for Evolving Business Activities," in Computing in the Global Information Technology, ICCGI'08. 2008, 277-283.

[23] M. Scheffer, F. Westley, W.A. Brock and M. Holmgren, "Dynamic Interaction of Societies and Ecosystems," in: L.H.S. Gunderson and C.S. Holling, q.v., 2002, 195-239.

[24] A. G. Tansley, "The use and abuse of vegetational concepts and terms," Ecology, vol 16, 1935, 284-307.

[25] B. Walker and D. Salt, Resilience Thinking, Island Press, 2006.

[26] World Commission on Environment and Development, Our Common Future, Cambridge University Press, UK, 1987. 